10.30972/eitt.704760

\title{
Coeficiente de Dilatación: diseño y construcción de un dilatómetro
}

Gabriel S. Pérez ${ }^{*}$, Ing. César A. Cabrera* ${ }^{*}$ Dr. Patricio F. Provasi*

\section{Resumen}

En prácticamente cualquier curso de termodinámica aparece el tema de dilatación, en particular la dilatación lineal. Pero también resulta de mucha importancia en la naturaleza ya que ésta se toma muy seriamente el tema al crear seres capaces de mantener constante su temperatura. Con la ayuda de materiales de descarte logramos realizar un dilatómetro que presenta una precisión muy alta y nos permite estimar el coeficiente de dilatación de, por ejemplo, el acero con una precisión inferior al $6 \%$.

Palabras claves: Termodinámica, Dispositivo de medición, Dilatación térmica, Dilatación lineal.

\section{Abstract}

In practically any course in thermodynamics, the topic of dilation comes up, particularly linear dilation. But it is also of great importance in nature since it takes the issue very seriously by creating beings capable of maintaining a constant temperature. With the help of discarded materials, we were able to make a dilatometer that has a very high precision and allows us to estimate the coefficient of 
expansion of, for example, steel with an accuracy of less than 6\%.

Keywords: Thermodynamics, Measuring device, Thermal expansion, Linear expansion.

\section{Introducción}

En el laboratorio de termodinámica de las carreras de Física, Química y casi todas las Ingeniería, normalmente aparece el tema de dilatación térmica, al menos una vez y aunque sea en su versión más elemental. Esto se debe, en gran medida a su importancia en todas estas áreas del conocimiento, pero también en que es una experiencia relativamente fácil de llevar a cabo. Así podemos encontrar una amplia gama de trabajos dedicados a la demostración de la ley de dilatación térmica lineal desde los métodos más elementales y algunos demostrativos como ser el de la pelota y el aro o utilizando dos reglas plásticas idénticas pero sumergiendo una en nitrógeno líquido [1-3] hasta los más elaborados y precisos de superposición de imágenes infrarrojas, interferometría o simplemente usando un láser para amplificar la dilatación producida [4-8]. Dos review [9 y 10], resumen adecuadamente el estado del arte en los métodos y tecnología disponibles actualmente.

También pueden verse experimentos hechos con aparatos comerciales especialmente diseñados para tal fin, como es el caso de los equipos PASCO [11] o bien el uso ingenioso de un sensor de movimiento utilizado para la observación del fenómeno de dilatación térmica lineal [12]. Un dispositivo remarcable, es el diseñado para enseñar dilatación térmica lineal a gente ciega [13].

Con relación a la importancia del tema en la naturaleza podemos mencionar algunos ejemplos elementales, como ser el hecho que la temperatura juega un rol importante como agente de erosión, así la constante dilatación y contracción que sufren los minerales a nivel del suelo ayuda a quebrar y pulverizar la roca; en los litorales rocosos a partir de latitudes templadas y hacia las frías el agua y su particular comportamiento de dilatación entre $4^{\circ} \mathrm{C} \mathrm{y} \mathrm{O}^{\circ} \mathrm{C}$ provocan el rompimiento de las rocas. Por otro lado, esta propiedad del agua, es la razón fundamental que si no se siguen los protocolos en el proceso de congelamiento de alimentos, las células de 
los mismos terminen rompiéndose, lo que repercute en sabores diferentes, al ser cocidos, que en los alimentos no congelados, normalmente se distingue una pérdida significativa del sabor.

En particular los procesos biológicos ocurren en condiciones controladas de presión, temperatura, $\mathrm{pH}$ y algunas concentraciones moleculares e iónicas como el caso de sodio o potasio y las concentraciones de dióxido de carbono y oxígeno entre otras. Y como podemos ver, la naturaleza no escatimó esfuerzos en llegar a producir organismos de "sangre caliente“, mamíferos incluido el hombre, los que mantenemos la temperatura prácticamente fija a un valor de aproximadamente $37^{\circ} \mathrm{C}$, a pesar que ello implique que la mayor parte de la Energía Basal, necesaria para mantener la vida, se disipe en forma de calor al medio ambiente.

De forma que el estudio del comportamiento de los materiales con los cambios de temperatura es de importancia, no solo en la física o en la mayoría de las ingenierías sino también en la bioquímica y biología, resultan de interés.

\section{Materiales y Métodos}

Usualmente el coeficiente de dilatación térmica longitudinal de cualquier material se define como el incremento de longitud por unidad de aumento de temperatura del material. Matemáticamente sería:

$$
\alpha=\frac{1}{L_{0}} \frac{\Delta L}{\Delta T}
$$

Pero esta forma matemática es válida en la parte de comportamiento aproximadamente lineal del mismo, y no tendría validez cuando la forma de la curva deja de ser lineal. Por ello, una alternativa es la de usar la definición en función de la derivada de la longitud en función de la temperatura.

Así, una ecuación más general sería

$$
d L=L \alpha \mathrm{dT}
$$

que tiene una integral

$$
L=L_{0} e^{\alpha \Delta T}
$$


y que puede reescribirse como la serie

$$
L=L_{0}\left(1+\alpha \Delta T+\frac{1}{2} \alpha^{2} \Delta T^{2}+\ldots\right)
$$

Sin embargo, para los rangos de temperaturas que podemos disponer en un aula o laboratorio lleno de adolescentes y sin correr riesgos de accidentes graves, va entre $\mathrm{O}^{\circ} \mathrm{C}$ y $100^{\circ} \mathrm{C}$, que son temperaturas que todos manejan en sus respectivos hogares.

En este rango de temperaturas los materiales comunes que disponemos en casa, exceptuando casos como el agua o la goma, en su mayoría, ya sean metales (hierro, bronce aluminio, cobre, etc.) o compuestos (cerámica, vidrio, porcelana, etc.) tienen un comportamiento aproximadamente lineal con la temperatura, Ec. (1).

Un punto de vista interesante de remarcar es el mencionado por Fakhruddin [14] que le muestra al alumno leyes físicas similares, tal es el caso de la expresión (3) y (4) y la ley de decaimiento radiactivo (o también la descarga de un condensador) y proponerle una situación como es el caso de la constante de decaimiento del Uranio (o una relación $1 / \mathrm{RC}$ pequeña) que permite reducir la serie al mismo caso habitualmente estudiado para la dilatación térmica.

Este último punto, la comparación de leyes físicas homomórficas, permiten economizar memoria y generalizar mecanismos que evidentemente son repetitivos en la naturaleza.

\section{Construcción del dispositivo}

Los materiales necesarios para el dilatómetro del presente trabajo son:

- Recortes de madera con los que se fabricó el banco de trabajo, el soporte de la muestra y el regulador de tope.

- Recorte de chapa de zinc para el Cuadrante.

- Un retazo de la corredera de un cajón de la alacena de la cocina, para la corredera.

- Tornillo milimetrado, con su respectiva tuerca para el tornillo micrométrico.

- Una escala impresa en papel. 
Figura 1: Fotos del dispositivo construido en diferentes ángulos y acercamientos para poder apreciar los detalles
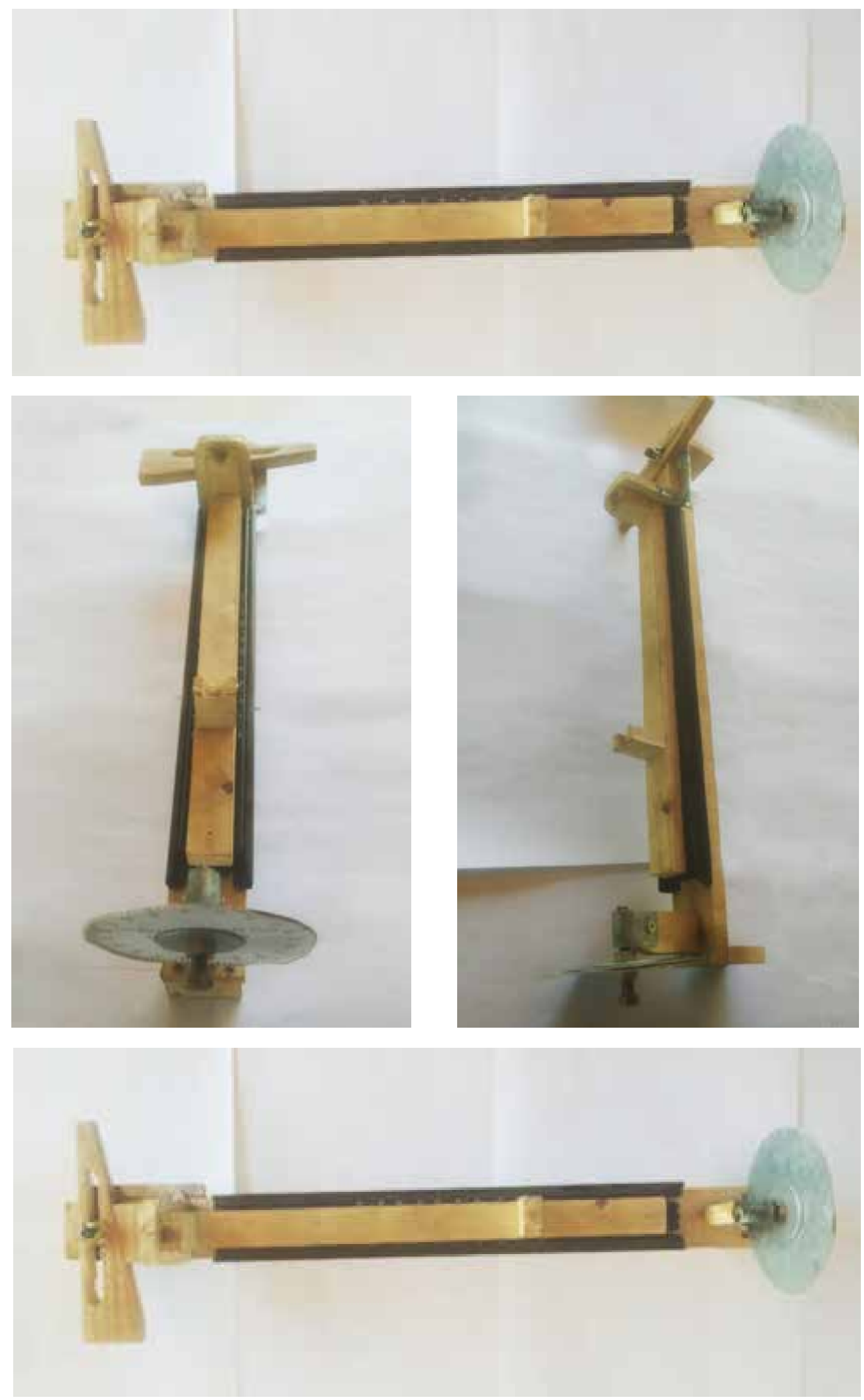
Esquema 1: Vistas del dilatómetro y detalle de sus partes.

\section{DILATÓMETRO}
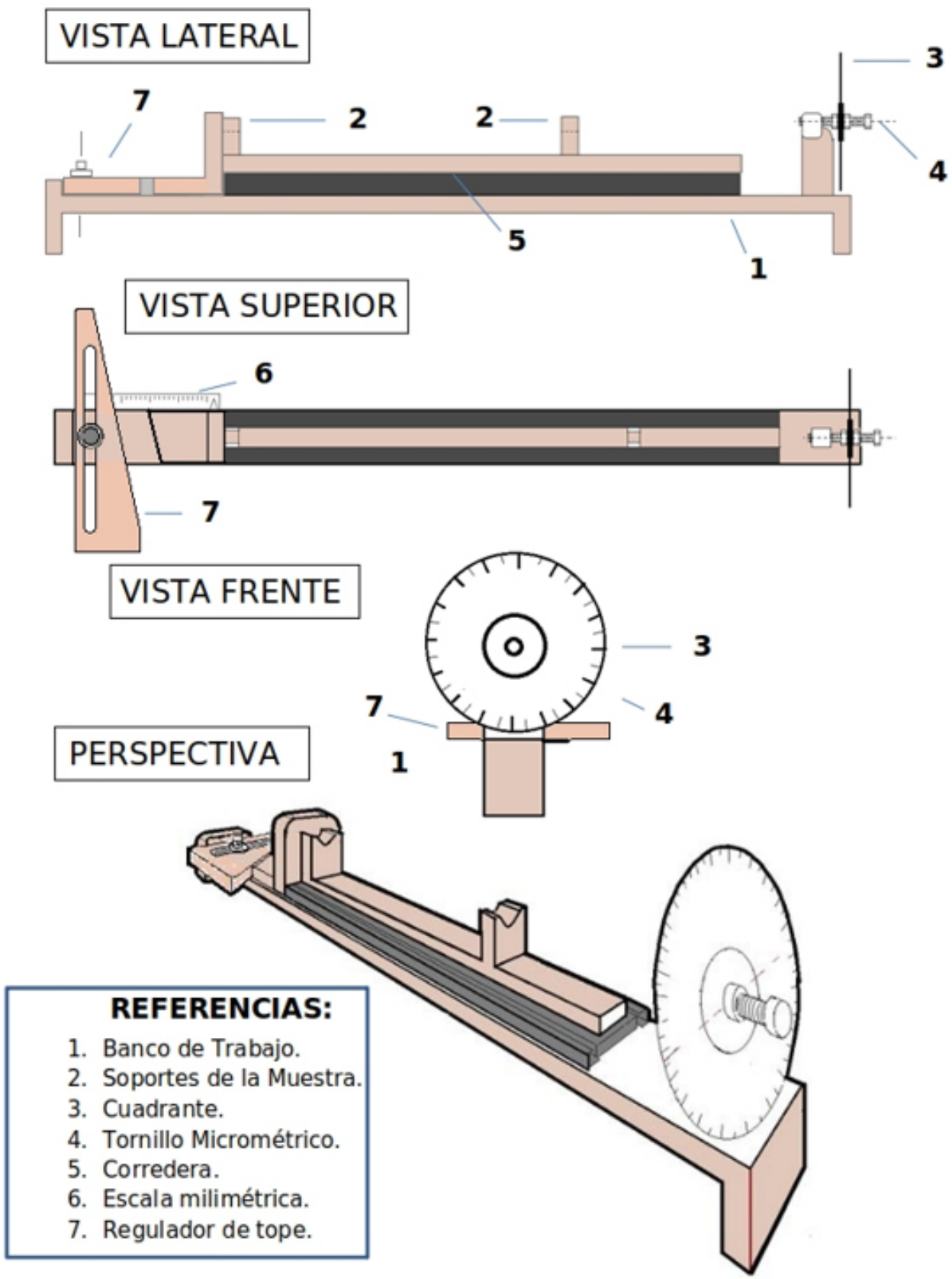


\section{Experimento}

Para el desarrollo del experimento se necesita además, una varilla del material que se desea conocer su coeficiente de dilatación lineal, un recipiente que contenga hielo y agua, y otro con el agua en ebullición, un sensor de temperatura para constatar los valores de $0^{\circ} \mathrm{C}$ y $100^{\circ} \mathrm{C}$ (si no se cuenta con él podría funcionar igualmente), un poco de papel absorbente para secar la varilla luego de estar sumergida en alguno de los recipientes mencionados y una pinza para extraer la varilla de los recipientes y manipularla.

Con este dispositivo se determinó para el acero un coeficiente de dilatación de:

$$
\alpha=\frac{\Delta L}{L_{0} \Delta T}=\frac{0,39}{326,61 \times(100-6)}=12,7 \times 10^{6}{ }^{\circ} \mathrm{C}^{-1}
$$

Dado que la longitud final (caliente), es de $327,00 \mathrm{~mm}$ a $100^{\circ} \mathrm{C}$ y final (fría) de $326,61 \mathrm{~mm}$ a $6^{\circ} \mathrm{C}$ (temperatura del agua de la heladera, controlado con un sensor de temperatura).

Comparando el valor experimental con el valor reportado para el acero (12,0 x $106{ }^{\circ} \mathrm{C}^{-1}$ ) obtenemos un error de $5,8 \%$.

El dilatómetro también admite la posibilidad de utilizar un cilindro con su base sellada que puede llenarse con un fluido, por ejemplo, aire o agua, y colocar un émbolo móvil, en el otro extremo. En estos casos sería necesario contar con un sensor de temperatura, ya que, en el caso de gases, debería considerarse una temperatura muy inferior a la de ebullición del agua. Podría ser, por ejemplo, la difusión del hielo y la del ambiente. En el caso de la dilatación de un líquido, sería necesario conocer el coeficiente de dilatación del material del cilindro que se utiliza para contenerlo y hacer la consabida corrección, pero para los gases no sería necesario.

\section{Conclusiones}

Los materiales de construcción son muy accesibles, de hecho se los podría conseguir como desperdicio en alguna carpintería especializada en colocación de muebles de cocina. 
La parte más sensible es el cuadrante y el tornillo de rosca milimétrica que logra funcionar como tornillo micrométrico gracias a la relación del diámetro del cuadrante y a las divisiones que se le pueden incorporar de acuerdo a su diámetro.

La precisión que se logra en la medición, como se puede ver de la sección anterior, es de una centésima de milímetro y considerando su bajo costo de construcción resulta un dispositivo sumamente conveniente.

\section{Agradecimientos}

Este trabajo se hizo posible mediante la financiación recibida a través del Proyecto PI 17/Foo8 Res 0966/17 CS. de la Secretaría General de Ciencia y Técnica (SGCyT) de la Universidad Nacional del Nordeste (UNNE).

\section{Bibliografía}

[1] (a) Peter Insley, Chis Chiaverina, and Jim Hicks "Thermal expansion plus" Phys. Teach. 22 (1984) 530-531. (b) S. K. Chakarvarti "Demonstration of the thermal expansion of solids" Phys. Teach. 26 (1988) 400. (C) R. Mostert "Classroom experiments on thermal expansion of solids" Phys. Teach. 30 (1992) 15. (d) $R$. Trumper and M. Gelbman "Measurement of a thermal expansion coefficient" Phys. Teach. 35, (1997) 437-438.

[2] Y. Kraftmakher "Two computer-assisted experiments" Phys. Educ. 48 (2013) 636-639.

[3] D. C. Pizetta e V. R. Mastelaro, Construção de um dilatômetro e determinação do coeficiente de dilatação térmica linear (Building a dilatometer and determining the coefficient of linear thermal expansion), Revista Brasileira de Ensino de Física, 36 (2014) 1313 (1-5).

[4] R. Scholl and B. W. Liby "Using a Michelson Interferometer to Measure Coefficient of Thermal Expansion of Copper" Phys. Teach. 47 (2009) 316-318. 
[5] R. Montanini and F. Freni "A new method for the determination of the coefficient of thermal expansion of solid materials" 11th International Conference on Quantitative InfraRed Thermography, (2012).

[6] Eriend H. Graf "A demonstration apparatus for linear thermal expansion" Phis. Teach. 50 (2012) 181.

[7] S. S. R. Inbanathan, K. Moorthy, and G. Balasubramanian "Measurement and Demonstration of Thermal Expansion Coefficient” Phys. Teach. 45 (2007) 566-567.

[8] H. Fakhruddin "Quantitative Investigation of Thermal Expansion Using Single-Slit Diffraction” Phys. Teach. 44 (2006) 82-84.

[9] J D James, J A Spittle, S G R Brown and R W Evans “A review of measurement techniques for the thermal expansion coefficient of metals and alloys at elevated temperatures" Meas. Sci. Technol. 12 (2001) R1-R15.

[10] M. Y. Hussain Ansari, M. W. Hussain, G. Lavanya, G. Rakesh and K. Ashok "Review on the Methods for the Measurements of Thermal Expansion Coefficient" J. App. Phys. 10 (2018) 47-50.

[11] J. N. Torres "Two Physical Phenomena in One Experiment" Phys. Teach. 44 (2006) 438.

[12] R. Gunderson "Motion Detectors Used in Measuring Heat Expansion" Phys. Teach. 56 (2018) 350-351.

[13] O Negrete, A Lisboa, F J Peña, C. O. Dib and P. Vargas "Teaching labs for blind students: equipment to measure the thermal expansion coefficient of a metal" Eur. J. Phys. 41 (2020) 035704 (1-9)

[14] H. Fakhruddin "Thermal expansion paradox" Phys. Teach. 31 (1993) 214. 